\title{
Detection of cytokine activated chondrocytes in arthritic joints from pigs infected with Erysipelothrix rhusiopathiae
}

Strangeways Research Laboratory, Worts Causeway, Cambridge CBI 4RN, United Kingdom M E Davies A Horner

Institute of Microbiology and Infectious Diseases, The Veterinary School, Bischofsholer Damm 15, D-3000 Hannover, Germany

B Franz

The Immunology Unit, The Veterinary School, Bischofsholer Damm 15, D-3000 Hannover, Germany

H-J Schuberth

Correspondence to:

Dr M Elisabeth Davies,

Tissue Physiology

Department,

Strangeways Research

Laboratory,

Worts Causeway,

Cambridge CBI 4RN,

United Kingdom.

Accepted for publication

10 March 1992

\author{
M Elisabeth Davies, Alan Horner, Burkart Franz, Hans-Joachim Schuberth
}

\begin{abstract}
Chronic polyarthritis was induced in pigs by injection of Erysipelothrix rhusiopathiae and the in vivo activation of chondrocytes by cytokines was then investigated in the affected joints by immunocytochemistry. A polyclonal antiserum which recognises surface markers on in vitro interleukin 1 activated porcine chondrocytes was used to detect activated chondrocytes in all zones of the cartilage from diseased joints. In contrast, cartilage removed from an unaffected joint in the same animal showed no chondrocyte activation. Inflammatory synovial tissue removed from diseased joints and cocultured with cartilage from the unaffected joint induced activation of adjacent chondrocytes. The presence of interleukin 1 in the inflammatory cells of the synovium was confirmed and major histocompatibility complex (MHC) class II antigens were detected as a marker of synovial activation. Chondrocytes were found not to express class II antigens in cartilage from either the diseased or the unaffected joint. These observations show that the porcine erysipelas model of arthritis will be useful in facilitating a novel approach to monitoring the behaviour of individual chondrocytes under pathophysiological conditions.
\end{abstract}

(Ann Rheum Dis 1992; 51: 978-982)

Rheumatoid arthritis is a chronic inflammatory joint disease of unknown aetiology which is characterised by pannus formation resulting in severe degeneration of the articular cartilage and bone.

Articular cartilage consists of chondrocytes embedded in an extracellular matrix made up of collagen fibrils and large aggregating proteoglycans. The dense fibrillar network provided by these matrix macromolecules is essential for maintenance of the structure and function of the cartilage and is controlled continuously by the activity of the chondrocytes. ${ }^{1}$ In healthy cartilage the role of these cells is to sustain a dynamic equilibrium between synthesis and degradation of these components.

Immune related disturbance of this balance in matrix turnover occurs in inflammatory joint diseases such as rheumatoid arthritis, eventually resulting in extensive depletion of the matrix constituents. The mechanisms involved in these degradative processes are, however, still unclear. Although it is generally accepted that the catabolic cytokines interleukin $1 \alpha$, interleukin $1 \beta$, and tumour necrosis factor $\alpha$ play a major part in chondrocyte induced cartilage destruction, ${ }^{23}$ the identity of the second messenger(s) remains to be determined. It is therefore of interest that high levels of tumour necrosis factor and interleukin 1 have been observed in the acute inflammatory response during the development of arthritis in Erysipelothrix rhusiopathiae infected rats. ${ }^{4}$

Alterations in the behaviour of chondrocytes in response to cytokine activation may well provide some useful clues to the processes taking part in joint damage in rheumatoid arthritis. Therefore, as a means of identifying cytokine responsive chondrocytes we raised a polyclonal antiserum which we have shown in vitro to be specific for interleukin 1 induced surface markers on pig chondrocytes. ${ }^{56} \mathrm{We}$ have now been able to extend our previous in vitro studies to investigate the behaviour of chondrocytes in vivo in pigs with chronic polyarthritis. The porcine erysipelas model has been extensively studied (for a review, see Schulz et $a l^{7}$ ) and is considered to be a satisfactory animal model of human rheumatoid arthritis. $^{8}$

The aim of this study was to locate sites of in vivo cellular activation induced by inflammatory cytokines produced during the disease process in this animal model.

\section{Materials and methods}

INFECTION OF ANIMALS

Five pigs (four to five months old) were experimentally infected by intraperitoneal injection of $5 \times 10^{6}$ colony forming units of Erysipelothrix rhusiopathiae Serovar 2 strain T28. This strain had been isolated from pigs with natural chronic erysipelas infection and was shown to be highly virulent. ${ }^{9} 10$ The experimentally infected pigs showed visible clinical signs of developing polyarthritis (stiffness, mild to moderate swelling of knee and metacarpophalangeal joints, and reluctance to move). Eight weeks after infection histological examination at necropsy showed typical arthritic inflammatory lesions in the affected joints. At this time synovium and cartilage explants were removed from the affected knee joints and were either used immediately for culture experiments or were frozen in OCT embedding compound (Miles) in liquid nitrogen and stored at $-70^{\circ} \mathrm{C}$ until used for histology. In one animal cartilage from an unaffected metacarpophalangeal joint was removed for use as a control cartilage.

\section{CLINICAL MONITORING}

The clinical status was evaluated by recording the body temperature as a marker of septicaemia. 
To monitor the arthritis an arthritic index was devised by assigning a value to the visible alterations of 1 (low degree of alterations such as redness, swelling, pain) or 2 (high degree of alterations) of each of the following parameters: general locomotion disorders (stiffness, reluctance to move, kyphosis, limping), arthritis of carpometacarpal joints, arthritis of tarsometatarsal joints, arthritis of elbow joint, arthritis of knee joint. Every animal could attain a maximum score of $5 \times 2=10$.

\section{COCULTURE OF NORMAL PIG ARTICULAR CARTILAGE WITH AUTOLOGOUS RHEUMATOID SYNOVIUM}

Cocultures were set up as described previously ${ }^{6}{ }^{11}$ except that rheumatoid-like synovium taken from the cartilage/pannus junction was used instead of synovial mince and cultures were not placed on grids and Millipore membranes. Cartilage explants were removed from an unaffected metacarpophalangeal joint of the same pig and were cocultured for 1, 2, 4, and 7 days and then frozen in liquid nitrogen as described previously. Cultures were carried out in Dulbecco's minimal essential medium (Gibco) containing $580 \mu \mathrm{g} / \mathrm{ml} \mathrm{L}$-glutamine, $120 \mu \mathrm{g} / \mathrm{ml}$ benzyl penicillin, and $200 \mu \mathrm{g} / \mathrm{ml}$ streptomycin, supplemented with $5 \%$ heat inactivated $\left(56^{\circ} \mathrm{C}\right.$ for 30 minutes) fetal calf serum. The culture medium was changed at two day intervals.

\section{ANTISERA}

A polyclonal antiserum was raised in rabbits and has previously been shown to be specific for interleukin 1 activated porcine chondrocytes. ${ }^{56}$ An antiserum raised against purified porcine interleukin $1 \beta$ was also used. ${ }^{12} A$ polymorphic mouse monoclonal antibody (B0191) was raised against bovine MHC class II antigen and shown to cross react with the porcine molecule. A polyclonal sheep antihuman factor VIII antigen was purchased from Serotec and screened for reactivity with porcine vascular tissue.

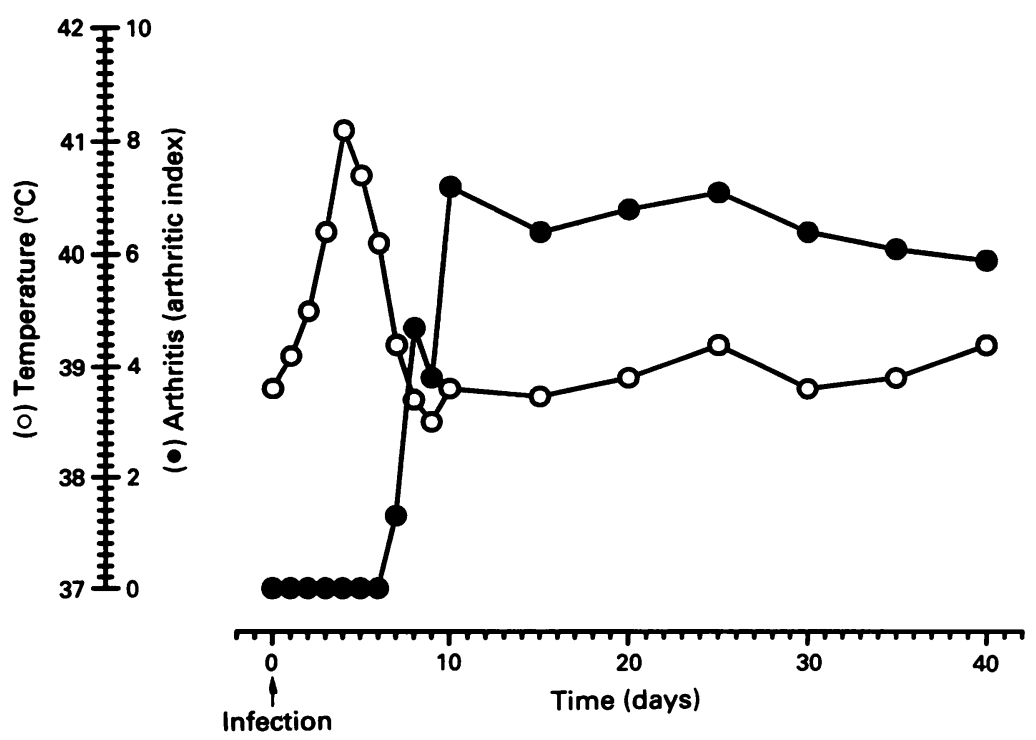

Figure 1 Development of body temperature changes $(O)$ and arthritis $(O)$ in pigs after infection with Erysipelothrix rhusiopathiae. For definition of the arthritic index see under Materials and methods. All data are mean values $(n=5)$.
All fluorescein (FITC) labelled secondary antisera, FITC swine antirabbit IgG, FITC rabbit antimouse IgG, and FITC rabbit antisheep IgG, were purchased from Dako (High Wycombe, Buckinghamshire, United Kingdom).

\section{IMMUNOHISTOLOGICAL TECHNIQUES}

Frozen sections 4-6 $\mu \mathrm{m}$ thick were cut at -25 to $-35^{\circ} \mathrm{C}$ onto poly-L-lysine (Sigma) coated eight well multitest slides (Flow Labs). Sections were air dried for 5-15 minutes then either fixed with $4 \%$ paraformaldehyde, $\mathrm{pH} 7 \cdot 4$, or stained unfixed within four hours. Unfixed sections were used for interleukin $1 \beta$ localisation; all other antisera were effective on fixed sections.

Sections were incubated with primary and secondary antisera essentially as described previously ${ }^{5}$ except that all antisera dilutions were made with $1 \%$ bovine serum albumin in phosphate buffered saline and for MHC class II localisation some sections were pretreated with hyaluronidase $(1 \mathrm{mg} / \mathrm{ml}$ for 15 minutes to maximise the exposure of antigen. Sections were viewed and photographed using the method described previously. ${ }^{6}$

Loss of proteoglycans from the cartilage matrix was assessed visually by toluidine blue staining of adjacent unfixed frozen sections. ${ }^{13}$

\section{Results}

\section{CLINICAL SYMPTOMS}

The first symptoms were obvious two days after infection when changes in the behaviour of the animal (malaise, general lethargy), an increase in body temperature, and signs of arthritis could be observed. The fever disappeared after one week but arthritic symptoms could be observed until the animals were killed (fig 1).

\section{ACTIVATION OF CHONDROCYTES IN ARTHRITIC JOINTS}

Animals which developed polyarthritis were killed eight weeks after infection with the organism. Dissected joints showed characteristic macroscopic lesions including swelling, increased amounts of viscous synovial fluid, extensive proliferation and vascularisation of the synovium, and pannus formation over a large area of the articular surface. At this stage the cartilage was not visibly eroded.

Immunohistological staining of the cartilage showed the expression of interleukin 1 activation antigens by most of the chondrocytes throughout the cartilage, from the articular surface to the hypertrophic zone. Chondrocytes in the articular and subarticular cartilage underlying the pannus showed particularly intense fluorescence (fig 2A). Midzone and hypertrophic chondrocytes showed fainter but more discrete membrane staining. Occasional non-activated chondrocytes were visualised by methyl green staining of the nuclei. The synovial inflammatory cells showed no immunoreactivity with the antiserum (fig 2A).

Invasion of the cartilage by inflammatory synovium was often observed. These infiltrating 

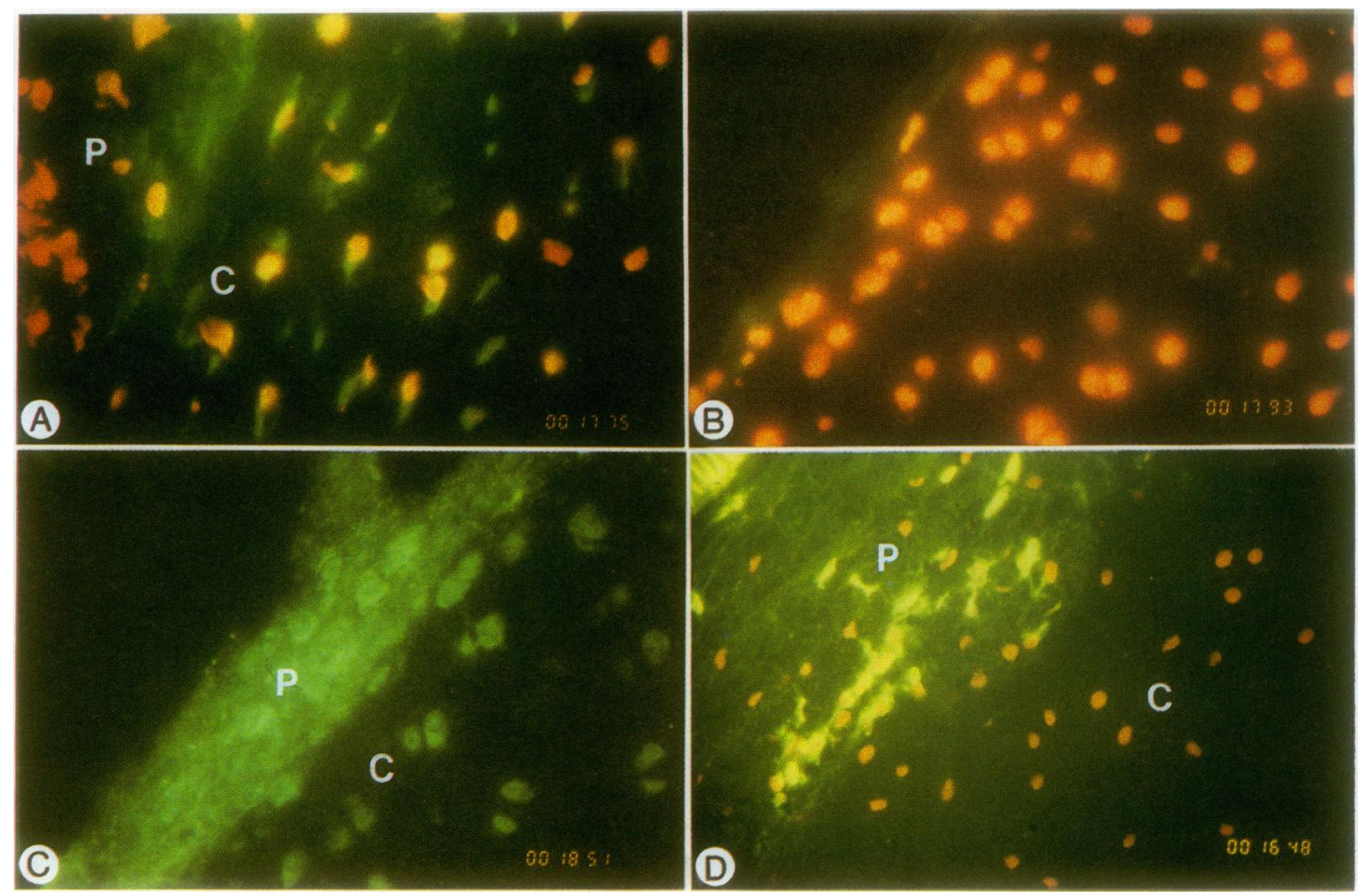

Figure 2 Immunolocalisation in frozen sections of cartilage and pannus from pigs infected with Ervsipelothrix rhusiopathiae. (A) Articular surface chondrocytes at cartilage $(C) /$ pannus $(P)$ junction. (B) Articular surface chondrocytes from an unaffected joint. (C) Immunolocalisation of porcine interleukin $1 \beta$, at cartilage $(C) /$ pannus $(P)$ junction. (D) Immunolocalisation of major histocompatibility complex (swine leucocyte antigen class II) antigen on the svnovial inflammatory cells of the pannus $(P)$. In sections $(A),(B)$, and $(D)$ nuclei were counterstained (red) to aid visualisation of non-reactive cells.

'fingers' consisted of closely packed inflammatory cells which were sometimes vascularised as confirmed by staining of the vessel endothelium with antiserum to factor VIII. Chondrocytes in the cartilage immediately surrounding these synovial infiltrates were also found to be strongly activated.

In contrast the normal unaffected joint showed no signs of swelling and dissection showed little synovial fluid, no synovial proliferation, and no pannus formation. Chondrocytes in the cartilage from these joints had no signs of activation and were detected only by their counterstained nuclei (fig 2B).

\section{MATRIX PROTEOGLYCAN DEPLETION}

Toluidine blue staining of non-fixed frozen sections of cartilage from the arthritic joint indicated trivial depletion of proteoglycans. Loss of metachromasia was seen only, in areas immediately adjacent to synovial tissue-that is, minimal depletion was observed at the articular surface beneath the pannus and in a narrow zone surrounding the inflammatory infiltrates (fig 3). This restricted depletion of proteoglycans presumably resulted from localised secretion of degenerative enzymes by the synovial cells. No loss of proteoglycan was seen surrounding individual chondrocytes in any region of the cartilage.

No loss of metachromasia was observed in cartilage from the unaffected joint.

ACTIVATION OF CHONDROCYTES IN NORMAL CARTILAGE BY AUTOLOGOUS INFLAMMATORY SYNOVIAL TISSUE

Although the chondrocytes in cartilage removed from the unaffected joint were not expressing activation markers, they were capable of being activated, as shown after coculture with autologous inflammatory synovium taken from an arthritic joint. Activation of the chondrocytes was observed after 24 hours of coculture. Maximum expression of activation markers was reached by $\mathbf{4 8}$ hours and was maintained during coculture for up to seven days. The presence of interleukin 1 in the inflammatory synovium from the arthritic joint was confirmed using a polyclonal antibody to porcine interleukin $1 \beta .^{12}$ The cytokine was localised in the matrix and in a high proportion of the cells of the synovial tissue (fig 2C). Some staining was also seen on the immediately adjacent chondrocytes.

\section{DISTRIBUTION OF MHC CLASS II EXPRESSION}

Major histocompatibility complex class II antigens, designated the swine leucocyte antigen complex $\mathrm{II},{ }^{14}$ were detected using a mouse polymorphic monoclonal antibody raised against bovine MHC class II antigens which has been shown to cross react with the porcine molecule (Schuberth, unpublished observations). In arthritic joints of five experimentally infected pigs we showed a strong expression of swine leucocyte antigen class II on all inflammatory synovial tissue, but were unable to detect the molecule on chondrocytes (fig 2D). The chondrocytes showed no green fluorescence and were visualised only by the methyl green staining of their nuclei. Pretreatment of sections with hyaluronidase had no effect on the distribution of swine leucocyte antigen class II expression.

A similar localisation was observed when normal pig cartilage was cocultured with inflammatory synovium from an arthritic joint. 


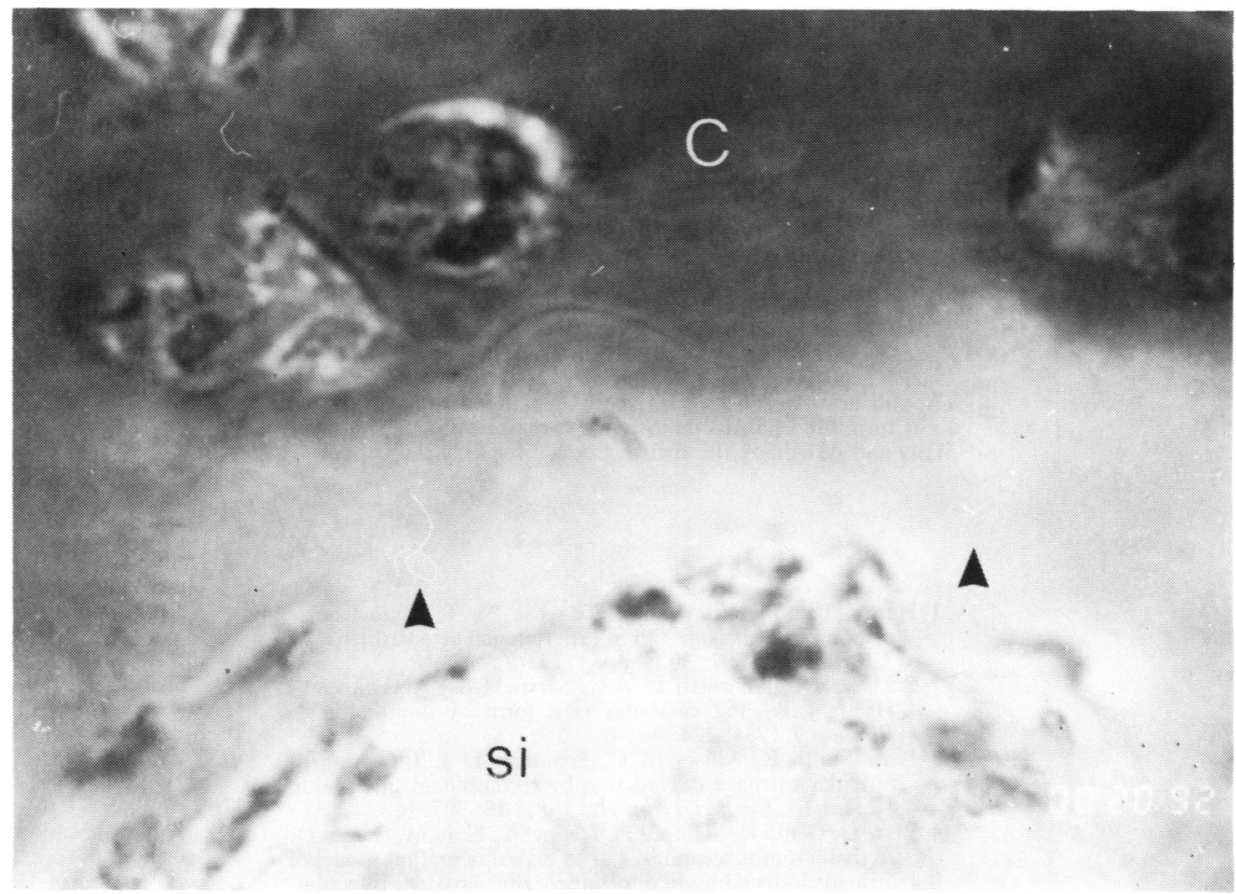

Figure 3 Toluidine blue staining of frozen sections of cartilage $(C)$ from pigs infected with Erysipelothrix rhusiopathiae showing (arrows) restricted loss of metachromasia (proteoglycan depletion) surrounding inflammatory synovial infiltrate (si) in mid zone cartilage.

Chondrocytes were activated by synovial mediators as already described but were not stimulated to express swine leucocyte antigen class II.

\section{Discussion}

We have developed a polyclonal antiserum which recognises phenotypic changes induced on pig chondrocytes in response to interleukin 1. Studies in vitro have already shown the usefulness of this antiserum to immunolocalise individual cytokine 'activated' chondrocytes in situ in cultured cartilage explants. ${ }^{56} \mathrm{We}$ also have available the porcine erysipelas polyarthritis animal model which has allowed us to use this antiserum to investigate activation of chondrocytes in vivo by cytokines secreted under pathophysiological conditions. An additional advantage of this model is that the disease progresses to a chronic self perpetuating autoimmune disease making it a suitable animal model for human rheumatoid arthritis. ${ }^{8}$

In this study we have identified activated chondrocytes in all samples of cartilage removed from arthritic joints of five pigs infected with Erysipelothrix rhusiopathiae. As activation was observed only in diseased cartilage and was most marked at the cartilage/pannus junction and surrounding synovial infiltrates, and in view of the specificity of the antiserum (Dingle and coworkers ${ }^{56}$ and unpublished observations) we assume that the chondrocytes in the diseased joints were activated in response to interleukin 1 secretion by the inflammatory synovium. Release of increased levels of cytokines including interleukin 1 has been reported in human rheumatoid synovium ${ }^{15} 16$ and we now confirm the presence of interleukin 1 in porcine inflammatory synovium.

From our observations we can conclude that at the particular stage of the disease studied in these experiments sufficient interleukin 1 had been produced in the arthritic joints to induce phenotypic changes in the chondrocytes in localised areas within the cartilage. Such changes may represent an early event, reflecting the response of the cells to the episodic or low level release of cytokines before the onset of persistent cytokine production $^{16}$ in the chronic stages of the disease. Certainly the integrity of the cartilage matrix in diseased joints, as assessed by minimal loss of metachromasia, suggests that the chondrocytes, though activated, were not yet in the process of destroying their surrounding extracellular environment.

We currently have little information on the nature of the surface markers induced by interleukin 1 and recognised by the polyclonal antiserum. The only well characterised cytokine 'activation' marker on chondrocytes so far reported is the MHC class II antigens. These molecules have been shown to be expressed in human rheumatoid arthritic cartilage ${ }^{17}$ and in response to the cytokine interferon $\gamma .{ }^{18}$ Class II antigens have also been reported on rabbit ${ }^{19}$ and rat $^{20}$ chondrocytes following isolation from normal articular cartilage. Although we showed expression of swine leucocyte antigen II (MHC class II) on the inflammatory synovial cells we were unable to detect this marker on the chondrocytes in cartilage from arthritic pigs. This result again implies limited cytokine production in the joints at this stage of the disease. Our findings so far, however, have encouraged us to undertake further experiments to study cytokine production and the activation of chondrocytes at different times during the progress of the disease to the chronic stage. In parallel we are raising monoclonal antibodies to make available additional markers of activated porcine chondrocytes. 
In conclusion our observations using the polyclonal antiserum in the erysipelas animal model have shown a novel way to simultaneously monitor cytokine production and chondrocyte behaviour during the development of arthritis. It is anticipated that this facility will provide new information to aid our understanding of the pathogenesis of inflammatory joint diseases such as rheumatoid arthritis.

The antiserum raised against purified porcine interleukin $1 \beta$ was a gift from Dr J Saklatvala (Strangeways Research Laboratory). MED and AH thank the British Council for financial support.

1 Hardingham T E, Venn G, Bayliss M T. Chondrocyte responses in cartilage and in experimental osteoarthritis. $\mathrm{Br}$ T Rheumatol 1991; 30 (suppl 1): 32-7.

2 Saklatvala J, Pilsworth L M C, Sarsfield S J, Gavrilovic J, Heath J K. Pig catabolin is a form of interleukin 1 . Biochem F 1984; 224: 461-6.

3 Lane Smith R, Allison A C, Schuman D J. Induction of articular cartilage degradation by recombinant interleukin $1 \alpha$ and 1B. Connect Tissue Res 1989; 18: 307-16.

4 Renz H, Gentz U, Schmidt A, Dapper T, Nain M, Gemsa D. Activation of macrophages in an experimental rat model of arthritis induced by Erysipelothrix rhusiopathiae infection. arthritis induced by Erysipelothrix

5 Dingle J T, Davies M E, Mativi B Y, Middleton H F. Immunohistological identification of interleukin-l activated chondrocytes. Ann Rheum Dis 1990; 49: 889-92.

6 Davies M E, Horner A, Dingle J T. Immunorecognition of chondrocytes in articular cartilage activated by synovial interleukin 1. Connect Tissue Res 1991; 25: 243-9.

7 Schultz L C, Ehard H, Hermanns W, et al. The different phases of Erysipelothrix polyarthritis: comparison with other microbial models. In: Deicher H, Schul\% L C, eds. Arthritis: models and mechanisms. Berlin, Heidelberg, New York: Springer Verlag, 1981.
8 Leibold W. Cellular immune reactions in Erysipelas polyarthritis in pigs. In: Deicher $\mathrm{H}$, Schuly L C, eds. Arthritis: models and mechanisms. Berlin, Heidelberg, New York: Springer Verlag, 1981.

9 Bisping W, Böhm K H, Weiland E. Zur derıeitigen Verlaufsform des chronischen Rotlaufs beim Schwein. III. Bakteriologische und serologische Ergebnisse. DTW Disch Tierarztl Wochenschr 1968; 75: 445-50.

10 Schulz L C, Drommer W, Seidler D, Erhard H, Leimbeck R, Weiss R. Experimenteller Rotlauf bei verschiedenen Spezies als Modell einer systemischen Bindegewebskrankheit. II Chronische Phase unter besonderer Berücksichtigung der Polyarthritis. Beiträge zur Pathologie 1975; 154: tigung

11 Fell $\mathbf{H}$, Jubb $R$ W. The effect of synovial tissue on the breakdown of articular cartilage in organ culture. Arthritis Rheum 1977; 20: 1359-71

12 Saklatvala J, Sarsfield S J, Wood D D. An antiserum to pig IL-1 (catabolin) reacts with the acidic but not the neutral form of IL-1. Br $\mathcal{F}$ R heumatol 1985; 24: 68-71.

13 Jubb $R$ W, Fell $H$ B. The breakdown of collagen by chondrocytes. I Pathol 1980; 130: 159-67.

14 Lunney J K, Pescovitz M D. Differentiation antigens of swine lymphoid tissue. In: Miyasaka $M$, Trinka $Z$ eds. Differentiation antigens in lymphohemopoietic tissues. New York: Marcel Dekker, 1988.

15 Firestein G S, Alvaro-Garcia J M, Maki R. Quantitative analysis of cytokine gene expression in rheumatoid arthritis. f Immunol 1990; 144: 3347-53.

16 Brennan F M, Field M, Chu C Q, Feldmann M, Maini R N. Cytokine expression in rheumatoid arthritis. Br.f Rheumatol 1991; 30 (suppl 1): 76-80.

17 Burmester GR, Menche D, Merryman P, Klein M, Winchester R J. Application of monoclonal antibodies to the characterization of cells eluted from human articular cartilage: expression of Ia antigens in certain diseases and identification of an $85 \mathrm{kD}$ cell surface molecule accumulated in the pericellular matrix. Arthritis Rheum 1983; 26: 1187-95.

18 Alsalameh S, Jahn B, Krause A, Kalden J, Burmester G R. Antigenicity and accessory cell function of human articular chondrocytes. F Rheumatol 1991; 18: 414-21.

19 Tiku M L, Lin S, Weaver C W, Teodorescu M, Skosey J L. Class II histocompatibility antigen-mediated immunologic function of normal articular chondrocytes. 7 Immunol 1985; 135: 2923-8.

20 Lance E M. Immunological reactivity towards chondrocytes in rat and man: relevance to autoimmune arthritis. Immunol Lett 1989; 21: 63-73. 